Original paper

\title{
Perspectives of hepatitis C virus (HCV) elimination in Poland
}

\author{
Robert Flisiak', Dorota Zarębska-Michaluk² \\ 'Department of Infectious Diseases and Hepatology, Medical University of Bialystok, Poland \\ ${ }^{2}$ Department of Infectious Diseases, Jan Kochanowski University in Kielce, Poland
}

\begin{abstract}
Aim of the study: According to the World Health Organization (WHO) strategy, elimination of hepatitis C virus (HCV) as a major public health threat by 2030 includes diagnosis and cure of $90 \%$ of those infected between 2015 and 2030. The aim of this study is to estimate the realistic possibility to achieve the WHO elimination targets in Poland.

Material and methods: The Polish population was established according to data from Statistics Poland for the year 2017. Treatment efficacy was estimated based on publications in time-frames depending on the availability of therapeutic options in Poland. The mortality of HCV-infected patients was assumed on the basis of Statistics Poland and the EpiTer-2 database The number of annual antiviral treatments was estimated based on data from the National Health Fund. Two scenarios were considered, without and with intervention dependent on the national screening program.

Results: The current diagnosis rate in Poland was calculated as 31\%. The scenario without intervention resulted in gradual reduction of annual treatments accompanied by a decrease in the number of infected patients in 2030 to $118000(0.31 \%$ of the current Polish population), which is only $38 \%$ compared with 2015 established as a baseline by WHO. Introduction of the HCV screening program, which includes 3 million people annually, would increase treatment uptake to 12000 per year and reduce the number of HCV-infected persons in Poland by $90 \%$.

Conclusions: The urgent implementation of the national screening program for HCV in Poland is essential to achieve the WHO goal by 2030. The screening strategy should include up to 3 million persons annually to achieve treatment uptake of 12 thousand patients per year.
\end{abstract}

Key words: epidemiology, HCV, screening, viral hepatitis.

Address for correspondence

Prof. Robert Flisiak, Department of Infectious Diseases and Hepatology, Medical University of Bialystok, 14 Żurawia St. 15-540 Białystok, Poland, e-mail: robert.flisiak@umb.edu.pl

\section{Introduction}

Hepatitis C virus (HCV) infection appears to be a major public health problem worldwide. It is currently estimated that hepatotropic viruses are responsible for the death of over a million people annually, making it comparable to the mortality from human immunodeficiency virus (HIV) infection, tuberculosis and malaria. According to recent epidemiologic reports, 71 million people are living with $\mathrm{HCV}$ infection worldwide.
Introduction of highly effective and safe direct acting antivirals (DAA) changed the landscape and perspectives of HCV treatment dramatically. In addition, this revolution, of a scale unprecedented in the history of medicine, has stimulated epidemiologic studies and encouraged research teams to create simulations and prognoses concerning hepatitis $\mathrm{C}$ and its consequences. Such projects have been implemented at various levels, starting with global, through regional, and national in cooperation with the Center for Disease Analysis (CDA) [1-3]. Such forecasting of the disease 
burden of chronic hepatitis $\mathrm{C}$ was also performed in Poland [4]. The study established 2013 as a baseline and considered the future burden of the disease using three possible scenarios to control or eliminate $\mathrm{HCV}$ in Poland, taking into account different treatment availability, effectiveness and annual diagnosis rates. In fact, during the following years the scenario closest to the most optimistic elimination option was applied in Poland. This scenario assumed an increase of treatment uptake to 10000 patients in 2017, which actually was even higher, reaching almost twelve thousand. In the following years the scenario assumed continuation at the stable level of 15000 patients treated annually, which was unfortunately impossible due to the lack of a national HCV screening strategy. As a result, in 2018 only 7000 patients were treated and no more were available from waiting lists. So, the forecast was predictive for the years 2015-2017 but it has failed since 2018. Moreover, this forecast did not include some factors affecting HCV epidemiology, which were unpredictable at the time of its construction, such as change in the age distribution of infected and treated patients.

In 2016, the World Health Organization (WHO) initiated an ambitious plan to eliminate viral hepatitis as a public health threat by diagnosing $90 \%$ of $\mathrm{HCV}$-infected subjects and treating $80 \%$ of all eligible patients by 2030 . The goal of the WHO is a reduction by $90 \%$ in HCV incidence and 65\% in HCV-related mortality $[5,6]$. The purpose of this study is to estimate the likelihood of achieving the WHO elimination targets in Poland.

\section{Material and methods}

Values of the selected parameters used for simulation and forecasting are shown in Table 1. The Polish population was established according to the most recent data from the Statistics Poland (SP; Główny Urząd Statystyczny) database for the year 2017. Treatment efficacy was estimated based on publications in timeframes depending on the availability of therapeutic options in Poland [7-13].

The mortality of HCV-infected patients for the period prior to the interferon-free (IFN-free) era was assumed to be $3 \%$ on the basis of SP data for age category corresponding to the average age of patients treated for HCV in Poland at that time. Due to obvious demographic changes observed in the treated population (lower median age, significant reduction of cirrhotics) in subsequent years we used the mortality rate estimated in the EpiTer-2 database [14]. For forecasting tasks this parameter was assumed to be constant after the year 2018 (Table 1).
The number of annual antivirals treatments in the period 2009-2015 was estimated on the basis of data from the National Health Fund (NHF). For the years 2016-2018 it was calculated based on the contract value and unit cost of every type of treatment option, maintaining the ratio of different regimens administered to patients from the EpiTer-2 database [14]. Projected data on number of treated patients were used to forecast after 2019 (Table 1).

Two scenarios regarding changes of the HCV-infected population in Poland by 2030 were considered for possible HCV elimination, without and with intervention dependent on the national screening program. The average annual screening and treatment rates needed to achieve the WHO target of reduction by $90 \%$ of the number of those infected in Poland were calculated based on the above parameters.

\section{Results}

Assuming that all diagnosed patients are or will be treated in the near future, the diagnosis rate was determined at $31 \%$. It was calculated as the difference between the number of infected patients in 2009 (230 000) set as a baseline point and 2018 (159 663).

The scenario without intervention considering no activities to implement the national HCV screening program in Poland assumed the projected reduction of treatment uptake every year. As shown in Figure 1

Table 1. Parameters used for simulation and forecasting

\begin{tabular}{|c|c|c|}
\hline \multicolumn{3}{|c|}{ Parameters } \\
\hline Number of residents & & 38422346 (SP, 30.06.2017) \\
\hline \multicolumn{3}{|l|}{ Treatment efficacy } \\
\hline $2009-2013$ & & $50 \%$ \\
\hline 2014 & & $60 \%$ \\
\hline 2015 & & $80 \%$ \\
\hline 2016-2018 & & $97 \%$ \\
\hline $2019-2030$ & & $99 \%$, projected \\
\hline \multicolumn{3}{|c|}{ Mortality rate of infected patients } \\
\hline $2009-2015$ & $3 \%$ & Based on demographic data from CSO \\
\hline 2016 & $1.6 \%$ & Based on EpiTer-2 data \\
\hline 2017 & $0.8 \%$ & \\
\hline $2018-2030$ & $0.5 \%$ & \\
\hline \multicolumn{3}{|c|}{ Number of treated patients } \\
\hline $2009-2015$ & & Data source - NHF \\
\hline 2016-2018 & Datas & urce - NHF, manufacturers and EpiTer-2 \\
\hline $2019-2030$ & & Projected data \\
\hline
\end{tabular}




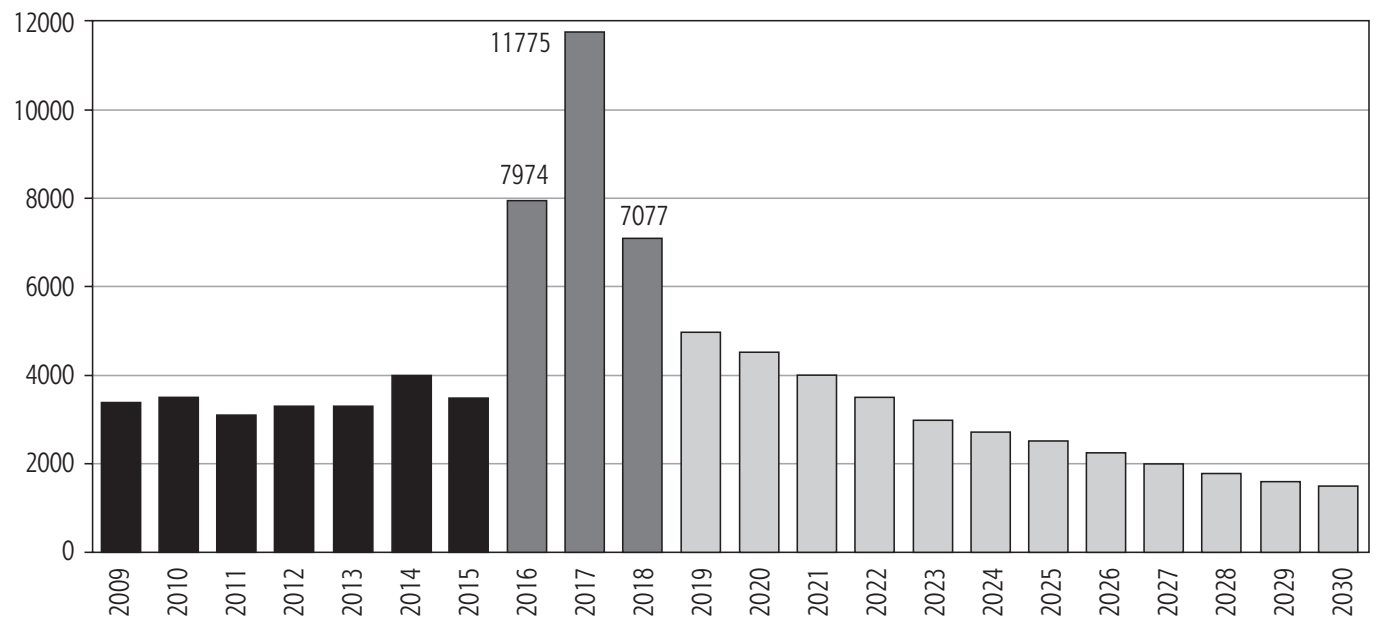

Fig. 1. Number of patients treated for HCV infection in 2009-2018 based on National Health Fund data (black bars), in years $2016-2018$ based on the value of the contract, sales and unit cost of therapy (data from National Health Fund and manufacturers) maintaining aspect ratio of different treatment regimens from EpiTer-2 (dark grey bars) and in 2019-2030 based on the forecast (light grey bars)

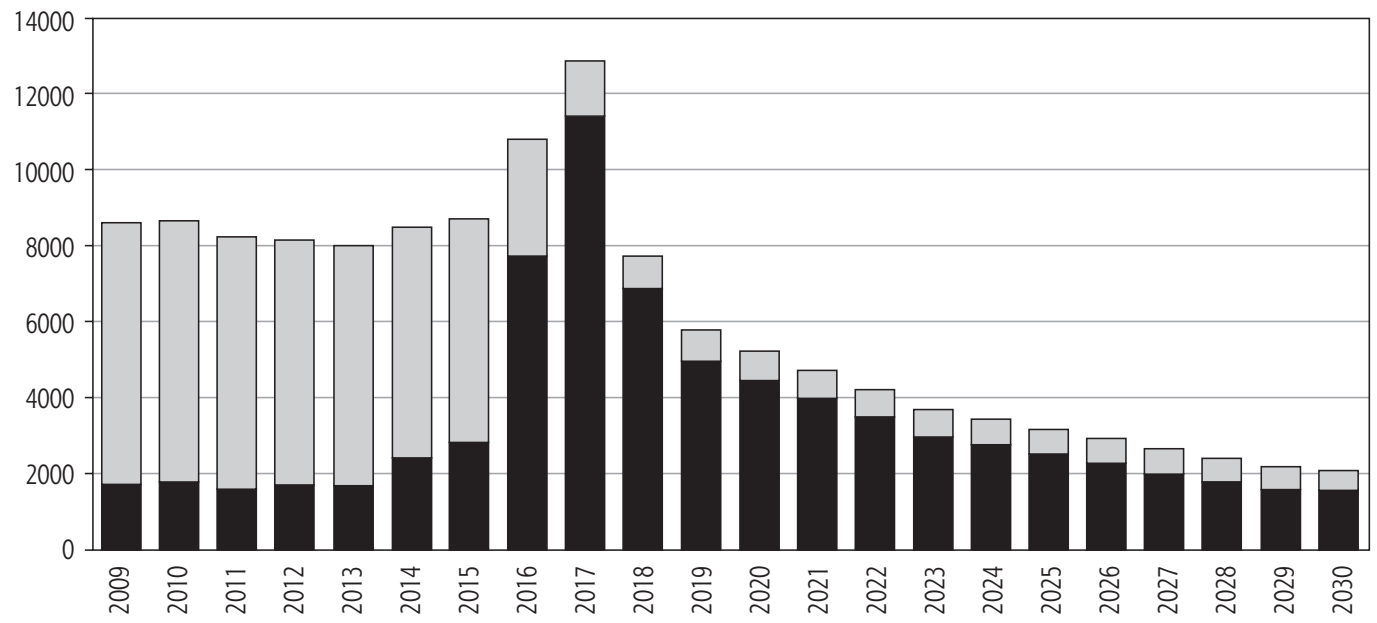

Fig. 2. HCV elimination in Poland in 2009-2030 including the number of patients cured (black bar) and died with HCV infection (grey bar)

the predicted number of annual DAA treatments in 2022 and subsequent years would be even lower than before 2016. Despite that, the number of infected patients would gradually decrease due to high treatment availability and its efficacy as well as mortality among elderly patients (Fig. 2). As a result, the number of infected patients would be reduced by 2030 to 118000 , corresponding to $0.31 \%$ of the current Polish population (Fig. 3). It means a nearly $50 \%$ reduction in the number of HCV-infected subjects compared with 2009 but only a 38\% decrease compared with 2015 established as a baseline by WHO. This calculation demonstrated no possibility to achieve a $90 \% \mathrm{HCV}$ diagnosis rate by 2030 using the scenario without intervention.

Since the introduction of a national screening program in Poland in 2019 is unlikely, we performed calculations assuming such intervention starting from 2020. We found that implementation of the HCV screening program would scale up the number of diagnosed patients and the projected treatment uptake could reach 12000 annually, giving over 167000 individuals treated in the years 2015-2030. This scenario would reduce the number of HCV-infected persons in Poland by an expected $90 \%$. Moreover, as shown in Figure 4, the predicted number of people living with HCV would decrease below 20000 by 2030, corresponding to $0.05 \%$ of the general Polish population. Assuming that the HCV incidence in Poland is $0.4 \%$, we calculated that the screening rate would need to be scaled up to 3 million persons per year to reach the projected annual treatment uptake of 12 thousand. 


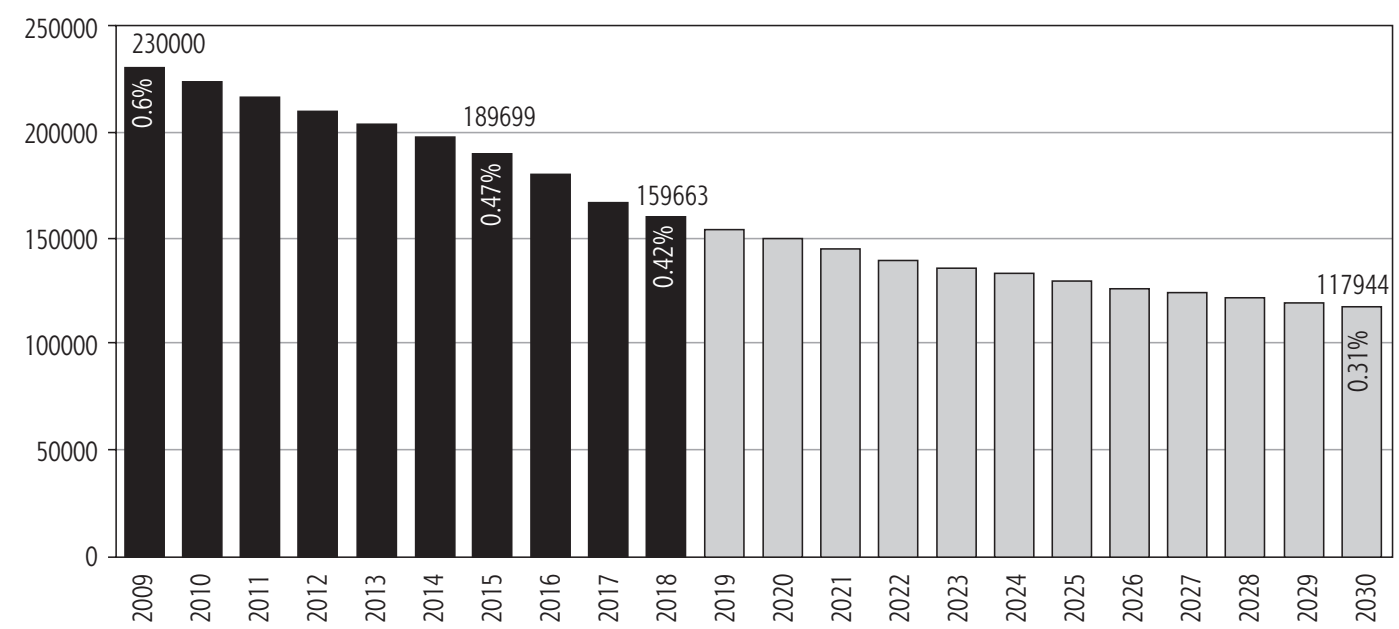

Fig. 3. Number of HCV-infected patients (HCV RNA positive) in 2009-2018, estimated on the basis of dynamics resulting from previous studies (black bars) and forecast 2019-2030 assuming no intervention in the form of the national screening program increasing the number of newly diagnosed patients (grey bars). The percentages on bars reflect the rate of infected patients in the general Polish population

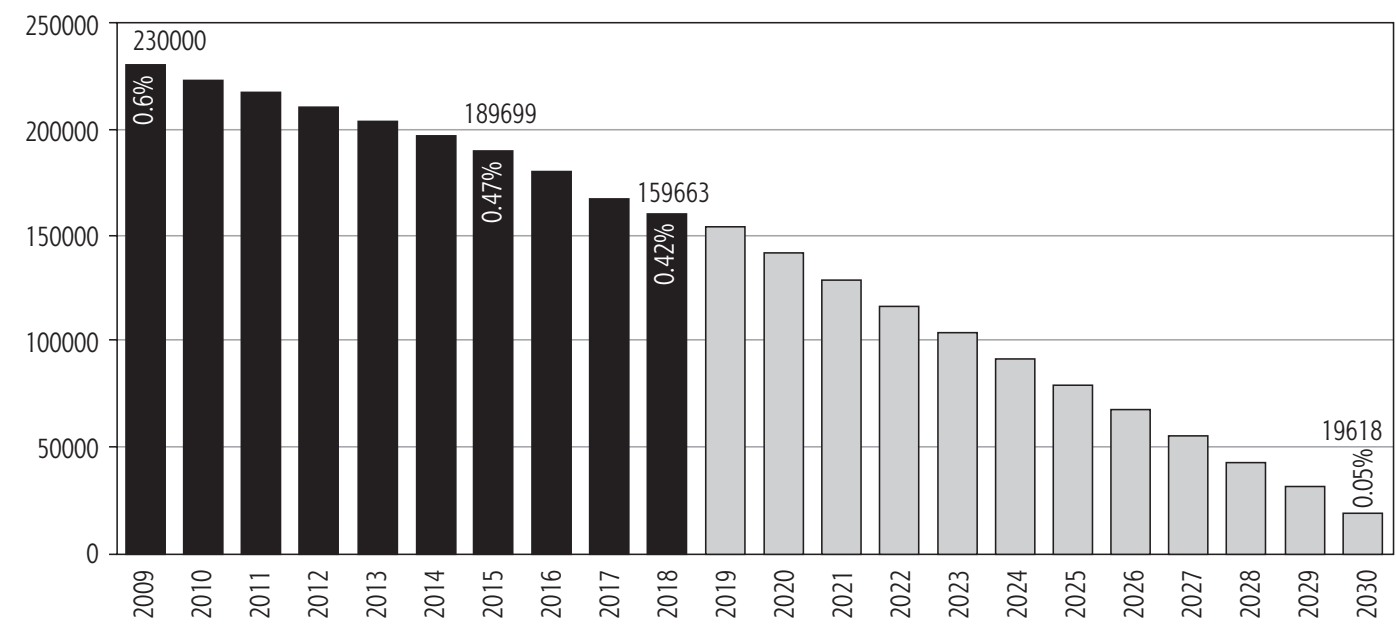

Fig. 4. Number of HCV-infected patients (HCV RNA positive) in 2009-2018, estimated on the basis of dynamics resulting from previous studies (black bars) and forecast 2019-2030 assuming an intervention in the form of the national screening program starting from 2020 and increasing number of newly diagnosed patients (grey bars). The percentages on bars reflect the rate of infected patients in the general Polish population

\section{Discussion}

Several epidemiological studies assessing HCV surveillance in Poland were conducted before 2010. However, they focused on small, unrepresentative populations and were limited to anti-HCV testing with a demonstrated rate of $1-2 \%$. Since we know that only $0.3-0.6 \%$ of individuals positive for the anti-HCV test are viremic, those results did not reflect actual HCV prevalence in the Polish population. However, one of them is worthy of special attention due to the large size of the tested cohort and long duration of assessment. The study was performed within 11 years in 61805 persons and found a downward trend in incidence of anti-HCV antibodies from $3.2 \%$ in 2004 to $1.1 \%$ in 2014 [15]. The first large scale epidemiologic study estimating the prevalence of active HCV infection (HCV RNA positive) in Poland was conducted by the Polish Group of Experts for HCV (PGE HCV) in the years 2009-2010. The study was performed in 26057 individuals, determining $0.6 \%$ incidence of viremic infections. It corresponded to 230 thousand Polish patients, mostly unaware of the disease and in need of antiviral treatment [16]. Another study completed in 2016 within a Swiss Contribution program confirmed $0.47 \%$ prevalence of HCV RNA in 21875 individuals [17]. The most recent not yet published screening study was carried out in a cohort of 300000 people in different populations and in various regions of Poland. The analysis revealed about $0.4 \%$ incidence of active HCV infections, corresponding to 150000 viremic patients needing to be diagnosed and treated in 
our country. So we assumed this HCV prevalence rate as a starting point to calculate the number of persons who ought to be tested.

It should be noted that the WHO strategy assumes as a goal elimination of viral hepatitis as a major public health threat but not global elimination of HBV and HCV infections [5, 6]. Furthermore, the WHO target is not $\mathrm{HBV}$ and HCV eradication in terms of removing these viruses from human organisms all over the world. The global eradication of infectious diseases is actually possible only by a successful mass vaccination program, as was the case with smallpox. Such a scenario is not attainable, because there is no anti-HCV vaccine available. However, the accessibility of increasingly affordable, highly effective antiviral regimens enables significant reduction of the HCV-infected population with a lower number of emerging infections and a relevant decrease of life-threatening consequences of the disease.

Based on the available data, it may be assumed that all diagnosed patients have access to DAA therapy in Poland. After passing a qualification procedure required by the NHF, all eligible individuals could be treated for HCV infection if they are interested in that. So we can assume that $80 \%$ of patients fulfilling the treatment criteria have access to antiviral therapy nowadays. Therefore, the diagnosis of at least $90 \%$ of $\mathrm{HCV}$ infections based on the forecast made in 2015 seems to be a key parameter required to reach the WHO elimination targets in Poland by 2030.

In contrast to previous forecasting reports, the current analysis included the mortality rate of HCV-infected patients, which is a very important factor affecting the number of patients living with $\mathrm{HCV}$, because the majority of them are over the age of 50 [13]. Based on results of studies published in 2011 and subsequent projections, we assumed that $20 \%$ of $\mathrm{HCV}$ patients were aware of their disease $[4,16]$. According to the current report, the diagnosis rate in the Polish $\mathrm{HCV}$-infected population should be established at the level of $31 \%$.

In conclusion, despite the wide access to highly effective treatment options, achievement of the WHO elimination targets in Poland is not attainable without a substantial scale-up of HCV testing. The urgent implementation of the national screening program is essential to achieve the WHO goal by 2030 . The screening strategy should include up to 3 million persons annually to achieve treatment uptake of 12 thousand patients per year.

\section{Disclosure}

The authors report no conflict of interest.

\section{References}

1. Cornberg M, Razavi HA, Alberti A, et al. A systematic review of hepatitis $C$ virus epidemiology in Europe, Canada and Israel. Liver Int 2011; 31 Suppl 2: 30-60.

2. Razavi H, Waked I, Sarrazin C, et al. The present and future disease burden of hepatitis $\mathrm{C}$ virus (HCV) infection with today's treatment paradigm. J Viral Hepatitis 2014; 21 Suppl. 1: 34-59.

3. Hatzakis A, Chulanov V, Gadano AC, et al. The present and future disease burden of hepatitis $\mathrm{C}$ virus (HCV) infections with today's treatment paradigm - volume 2. J Viral Hepat 2015; 22 Suppl 1: 26-45.

4. Flisiak R, Halota W, Tomasiewicz K, et al. Forecasting the disease burden of chronic hepatitis C virus in Poland. Eur J Gastroenterol Hepatol 2015; 27: 70-76.

5. WHO. Global hepatitis report, 2017. Geneva, Switzerland: World Health Organization. http://apps.who.int/iris/bitstream /10665/255016/1/9789241565455-eng.pdf (access: 7.04.2019).

6. WHO. Global health sector strategy on viral hepatitis 20162021. Geneva, Switzerland: World Health Organization. http:// apps.who.int/iris/bitstream/10665/246177/1/WHO-HIV2016.06-eng.pdf (access: 7.04.2019).

7. Flisiak R, Pogorzelska J, Berak H, et al. Efficacy of HCV treatment in Poland at the turn of the interferon era - the EpiTer study. Clin Exp Hepatol 2016; 2: 138-143.

8. Jacobson I, Zeuzem S, Flisiak R, et al. Daclatasvir vs telaprevir plus peginterferon alfa/ribavirin for hepatitis $\mathrm{C}$ virus genotype 1. World J Gastroenterol 2016; 22: 3418-3431.

9. Janczewska E, Flisiak R, Zarebska-Michaluk D, et al. Effect of peginterferon or ribavirin dosing on efficacy of therapy with telaprevir in treatment-experienced patients with chronic hepatitis $\mathrm{C}$ and advanced liver fibrosis: a multicenter cohort study. Medicine 2015; 94: e1411.

10. Flisiak R, Łucejko M, Mazur W, et al. Effectiveness and safety of ledipasvir/sofosbuvir \pm ribavirin in the treatment of HCV infection: The real-world HARVEST study. Adv Med Sci 2017; 62: 387-392.

11. Zarębska-Michaluk D, Flisiak R, Jaroszewicz J, et al. Is interferon-based treatment of viral hepatitis $\mathrm{C}$ genotype 3 infection still of value in the era of direct-acting antivirals? J Interferon Cytokine Res 2018; 38: 93-100.

12. Flisiak R, Janczewska E, Wawrzynowicz-Syczewska M, et al. Real-world effectiveness and safety of ombitasvir/paritaprevir/ ritonavir \pm dasabuvir \pm ribavirin in hepatitis C: AMBER study. Aliment Pharmacol Ther 2016; 44: 946-956.

13. Flisiak R, Zarębska-Michaluk D, Janczewska E, et al. Treatment of HCV infection in Poland at the beginning of the interferon-free era-the EpiTer-2 study. J Viral Hepat 2018; 25: 661-669.

14. Flisiak R, Lorenc B, Klapaczyński J, et al. Characteristics of patients and effectiveness of chronic hepatitis $\mathrm{C}$ treatment during the initial 4 years of access to interferon-free therapy. J Hepatology 2019; 70, Suppl 1: E222-E223.

15. Walewska-Zielecka B, Religioni U, Juszczyk G, et al. Anti-hepatitis $C$ virus seroprevalence in the working age population in Poland, 2004 to 2014. Euro Surveill 2017; 22: pii: 30441.

16. Flisiak R, Halota W, Horban A, et al. Prevalence and risk factors of HCV infection in Poland. Eur J Gastroent Hepatol 2011; 23: 1213-1217.

17. Rosińska M Parda N, Kołakowska A, et al. Factors associated with hepatitis $C$ prevalence differ by the stage of liver fibrosis: A cross-sectional study in the general population in Poland, 2012-2016. PLoS One 2017; 12: e0185055. 